S. E. Graversen and M. Rao

Nagoya Math. J.

Vol. 100 (1985), 163-180

\title{
QUADRATIC VARIATION AND ENERGY
}

\author{
S. E. GRAVERSEN AND M. RAO
}

\section{§ 0. Introduction}

It is well known that the concept of energy has played a fruitful role in potential theory and Markov processes. Cartan's work [6] led to kernel-free potential theories of Beurling-Deny [2]. Since then many authors have worked on this, M. Fukushima [8], M. Silverstein [16], J. Bliedner [3], Berg-Forst [1], to name some. In these works, however, the main thrust is Hilbert space theoretic.

In the martingale context the notion of energy was introduced by Meyer [11]. This has been applied in the potential theory of general Hunt processes in Z. R. Pop-Stojanovic and M. Rao [14], S. E. Graversen and M. Rao [10].

In a different direction G. Brosamler [5] in one original paper initiated the study of quadratic variation in the context of potential theory. Brosamler's formula suggests an intimate connection with energy and is indeed the inspiration for the present work.

Let us briefly describe the results. In Section 1 we define what we mean by the energy of a cadlag process, i.e. a right continuous process with left limits. Then we show that such a process is a sum of a martingale and a previsible process with a special "orthogonality to martingales" property. Uniqueness of this decomposition, its relation to standard decompositions and an Itô formula for $C^{1}$-functions are then discussed. In this connection we mention the work of Föllmer [9].

Section 2 deals with energy of potentials in the context of Hunt processes. There are at least two different definitions to be found in the literature [8, 14]. We discuss the relation of these with the energy of the process obtained by composing the process with the potential.

Section 3 deals with application of the preceding to classical potentials in bounded domains of the Euclidean space. We obtain the well-

Received September 3, 1984. 
known decomposition of $f(B)$ where $f$ is a BLD function and $B$ the Brownian motion as the sum of a martingale and a process of zero quadratic variation [8]. We close this work with a Brownian motion characterization of local BLD functions in terms of the notions introduced in Section 1 and Section 2.

$\S 1$.

\section{Notation}

$(\Omega, \mathscr{F}, P)$ will denote a probability space with a filtration $\mathscr{F}_{t}, 0 \leqq t$ $<\infty$ satisfying the usual conditions and free of times of discontinuity.

For an adapted process $X$ and an integer $n$ we set

$$
\Delta(n, i, X)=X_{(i+1) / 2^{n}}-X_{i / 2^{n}} \quad i \geqq 0
$$

and for square integrable processes $X, Y$,

$$
S(n, X, Y)=E\left[X_{0}, Y_{0}\right]+\sum_{i} E[\Delta(n, i, X) \Delta(n, i, Y)] .
$$

We will say that a process $X$ has finite energy if the set of numbers $S(n, X)=S(n, X, X)$ is bounded. We put

$$
\|X\|_{e}^{2}=\sup _{n} S(n, X)
$$

and

$$
Q(X)=\lim \sup _{n} S(n, X) .
$$

The set of adapted cadlag processes of finite energy is clearly a linear space.

Even under such general conditions we have the following

Theorem 1. Let $X$ be cadlag and of finite energy. Then $X$ has a decomposition

$$
X=M+A
$$

where $M$ is a martingale and $A$ is previsible. $A$ has the property: there is a subsequence $n_{j}$ such that for every square integrable martingale $g$

$$
\lim _{j} S\left(n_{j}, A, g\right)=0 .
$$

If $N+B$ is another such decomposition, then $A-B$ is a continuous martingale. 
Proof. Write

$$
\delta(n, i, X)=X_{(i+1) / 2^{n}}-E\left[X_{(i+1) / 2^{n}} \mid \mathscr{F}_{i / 2^{n}}\right]
$$

and

$$
M(n)=X_{0}+\sum_{i} \delta(n, i, X)
$$

$X$ being of finite energy, $M(n)$ is a well defined bounded sequence in $L^{2}$. Let $M(\infty)$ be a weak limit of $M(n)$ along a sequence $n_{j}$. Let $M$ be the cadlag version of the martingale determined by $M(\infty)$. Put

$$
A=X-M \text {. }
$$

Then $A_{0}=0$ and $A$ is cadlag. It is completely routine to show that, for each square integrable martingale $g$,

$$
g_{t}=E\left[g_{\infty} \mid \mathscr{F}_{t}\right],
$$

we have

$$
\lim _{n_{j}} \sum_{i} E\left[A_{(i+1) / 2^{n_{j}}} \Delta\left(n_{j}, i, g\right)\right]=\lim _{n_{j}} S\left(n_{j}, A, g\right)=0 .
$$

Let $T$ be any inaccessible stopping time, $B$ the increasing process

$$
B_{t}=1_{T \leqq t}
$$

and $C$ the continuous increasing process that $B-C$ is a martingale. From (4) we get

$$
E\left[\int_{0}^{\infty} A_{t} d\left(B_{t}-C_{t}\right)\right]=0
$$

Because $C$ is continuous and $A$ cadlag,

$$
E\left[\int_{0}^{\infty} A_{t} d C_{t}\right]=E\left[\int_{0}^{\infty} A_{t_{-}} d C_{t}\right]=E\left[\int_{0}^{\infty} A_{t_{-}} d B_{t}\right]
$$

because $C$ is the predictable dual projection of $B$. Using this in (5), we get

$$
E\left[\left(A_{T}-A_{T_{-}}\right): T<\infty\right]=0
$$

which evidently implies that $A$ cannot have a jump at an inaccessible stopping time. Referring to p. $88 C$ of [7], $A$ has to be previsible.

The second statement is clear because a previsible martingale has to be continuous. 


\section{Relation to standard decompositions}

It is natural to inquire what relation the decomposition given by Theorem 1 has to standard decompositions. Suppose $X$ is a cadlag semimartingale in the sense that $X=M^{\prime}+A^{\prime}$ where $M^{\prime}$ is a uniformly integrable martingale and $A^{\prime}$ is a process of integrable variation. By [12] $X$ is a special semimartingale provided it has finite energy as defined above. Then it is known that we may write $X=M+A$, where $A$ is previsible of integrable variation. We claim that this is the decomposition given by Theorem 1 . Indeed, let $X=N+B$ be a decomposition given by Theorem 1 . Let $g$ be a bounded martingale. Since $A$ is of integrable variation, for every $n, S(n, A, g)$ makes sense and tends to zero as $n$ tends to infinity because $A$ is previsible. It follows that

$$
\lim _{n} S(n, X, g)=\lim _{n} S(n, M, g)=E[M(\infty) g(\infty)] .
$$

But by definition of $N$, along a subsequence $n_{j}$,

$$
\lim _{j} S\left(n_{j}, X, g\right)=E[N(\infty) g(\infty)]
$$

Comparing this with the above, we conclude $M=N$ and hence $A=B$.

\section{Uniqueness of the decomposition}

We now discuss when there is uniqueness in the decomposition given by Theorem 1 .

Corollary 2. Let $X$ be as in Theorem 1. Suppose for some decomposition

$$
X=M+A
$$

given by Theorem $1, Q(A)=0$. Then there is uniqueness in Theorem 1 .

Proof. Suppose $X=N+B$ is another decomposition. As observed in Theorem $1, A-B=W$ is a continuous martingale. There is a subsequence $n_{j}$ such that

$$
\lim _{j} S\left(n_{j}, B, g\right)=0
$$

for all square integrable martingales g. In particular,

$$
0=\lim _{j} \sup S\left(n_{j}, A\right)=E\left[W^{2}(\infty)\right]+\lim _{j} \sup S\left(n_{j}, B\right) .
$$

We must have $W=0$ and hence $A=B$ 
Now it is clear that $\|X\|_{e}$ is a norm. Convergence in this norm will be termed convergence in energy. If $Q(X)=0$, we shall say that $X$ has quadratic variation zero. It is well known that if $B$ is the Brownian motion in a bounded domain and $f$ is BLD, then $f(B)$ is the sum of a martingale and a process of zero quadratic variation. This is the motivation for the following theorem.

Let $\mathscr{H}$ denote the space of cadlag processes of finite energy which can be written as a sum of a square integrable martingale and a process of quadratic variation zero. $\mathscr{H}$ is a normed space with energy norm.

Theorem 3. Let $X \in \mathscr{H}$. Then the decomposition given by Theorem 1 is unique. Semimartingales as defined in Meyer [12] are dense in $\mathscr{H}$. If $X$ is cadlag and if there exists a sequence $X^{k} \in \mathscr{H}$ such that

$$
\lim _{k} Q\left(X-X^{k}\right)=0
$$

then $X \in \mathscr{H}$. If the corresponding decompositions are $M^{k}+A^{k}, M+A$, then also

$$
\lim _{k} Q\left(M-M^{k}\right)=0 .
$$

Proof. For the first part, note that if $Y$ is cadlag, has finite energy and $Q(Y)=0$, then the martingale part of the decomposition given by Theorem 1 is 0 -this is seen for example as in the proof of Corollary 2. In particular, $Y$ is previsible-indeed $Y$ is continuous as is easily seen, see the proof of Proposition 4. To prove the density part, let

$$
X=M+A
$$

where $Q(A)=0$. Define for $n=1,2, \cdots$

$$
A_{n}(t)=A\left(\frac{i}{2^{n}}\right) \quad \text { if } \frac{i}{2^{n}} \leqq t<\frac{t+1}{2^{n}}
$$

and $X_{n}=M+A_{n}$.

Then $X_{n}$ is a semimartingale, and since $S\left(k, A-A_{n}\right)=0$ for $k \leqq n$,

$$
\left\|X-X_{n}\right\|_{e}^{2} \leqq 2 \sup _{m \geqq n} S(m, A)
$$

and the last term tends to zero because $A$ has zero quadratic variation. The proof of the last assertion is just as easy. 


\section{Stopped processes}

Proposition 4. Let $X$ be cadlag and have finite energy. Let $T$ be a stopping time such that

$$
\sup _{t \leqq T} X_{t}
$$

is square integrable. Then the stopped process $Y$ defined by

$$
Y_{t}=X_{T \wedge t}
$$

also has finite energy.

Proof. For any stopping time $S$

$$
E\left[\left(X_{S}-X_{S-}\right)^{2}\right] \leqq \sup _{n} S(n, x) .
$$

Indeed, the left side above is less or equal to

$$
\lim _{n} \inf \sum_{i} E\left[\Delta(n, i, X)^{2}: \frac{i}{2^{n}}<S \leqq \frac{i+1}{2^{n}}\right] \leqq\|X\|_{e}^{2} .
$$

Therefore, for any $n$

$$
\begin{aligned}
S(n, Y)= & \sum_{i} E\left[\Delta(n, i, X)^{2}: i+1<2^{n} T\right] \\
& +\sum_{i} E\left[\left(X_{T}-X_{i 2-n}\right)^{2}: i<2^{n} T \leqq i+1\right] .
\end{aligned}
$$

The first term on the right side above is less or equal to $S(n, X)$. And

$$
E\left[\left(X_{T}-X_{i 2-n}\right)^{2}: i<2^{n} T \leqq i+1\right] \leqq 2 E\left[X_{T}^{2}+\left(X_{T}^{*}\right)^{2}: i<2^{n} T \leqq i+1\right]
$$

where

$$
X_{T}^{*}=\sup _{t \leqq T} X_{t} .
$$

Proposition 6. Let $X, Y, T$ be as in Proposition 4. If $X=M+A$ is a decomposition of the type given in Theorem 1 , then

$$
Y=M^{T}+A^{T}
$$

is a decomposition of $Y$ of the type considered.

The proof is routine and is omitted.

\section{$C^{1}$-Itô formula}

Recall that $\mathscr{H}$ denotes the space of cadlag processes of finite energy which can be written as a sum of a square integrable martingale and a 
process of quadratic variation zero. We then have the following theorem:

Theorem 6 (Itô formula). Let $X=M+A$ be continuous and belong to $\mathscr{H}$. Let $f$ be continuously differentiable with bounded derivative. $Y=$ $f(X) \in \mathscr{H}$ and the martingale part of $Y$ is

$$
Y_{0}+\int_{0}^{t} f^{\prime}\left(X_{s}\right) d M_{s} .
$$

Proof. Recall the notions $S, \Delta$ introduced in the begining and in the proof of Theorem 1. Since $f$ has bounded derivative, $Y$ is of finite energy. Let us prove that the sequence

$$
Y_{0}+\sum_{i} \delta(n, i, Y)
$$

converges weakly in $L^{2}$. This will be the martingale part of $Y$. We have

$$
\delta(n i, Y)-f^{\prime}\left(X_{2-n i}\right) \delta(n, i, X)=Z_{i, n}-E\left[Z_{\imath, n} \mid \mathscr{F}_{i 2-n}\right]
$$

where

$$
Z_{i, n}=\int_{\left[X_{i 2}-n, X^{\prime}(i+1) 2-n\right]}\left[f^{\prime}(y)-f^{\prime}\left(X_{i 2-n}\right)\right] d y .
$$

Since $f^{\prime}$ is bounded and $X=M+A$ with $Q(A)=0$, we have

$$
\lim _{n} \sum_{i} f^{\prime}\left(X_{2-n_{i}}\right) \delta(n, i, X)=\int_{0}^{\infty} f^{\prime}\left(X_{s}\right) d M_{s}
$$

where the limit in (9) is in probability and in $L^{2}$. Thus to prove the Theorem it is sufficient to show that

$$
\lim _{n} \sum E\left[Z_{i, n}^{2}\right]=0 .
$$

Since $X=M+A, Q(A)=0$ and $\sum \Delta(n, i, M)^{2}$ is uniformly integrable by [12], we also have that $\sum_{i} \Delta(n, i, X)^{2}$ is uniformly integrable. Since $f^{\prime}$ is bounded, the same is true of $\sum_{i} Z_{i, n}^{2}$. Also since

$$
\sum_{i \geq R 2^{n}} \Delta(n, i, X)^{2} \leqq 2 E\left[(M(\infty)-M(R))^{2}\right]+2 S(n, A)
$$

which is small if $R$ and $n$ are large, it is sufficient to show that

$$
\sum_{i \leq 2^{n} R} E\left[Z_{i, n}^{2}\right]
$$

is small if $n$ and $R$ are large. This last follows easily from the assumptions that $X$ and $f^{\prime}$ are continuous, $f^{\prime}$ bounded and the uniform inte- 
grability of $\sum_{i} \Delta(n, i, X)^{2}$ which has already been shown.

That proves the Theorem.

Remark. If $X$ is uniformly bounded, we do not need to assume $X$ is continuous.

\section{§2. Application to excessive functions}

Let $X$ be a transient Hunt process on a loc. compact space E. By this we mean that the last exit time from any compact set is finite almost surely. The notation and terminology will be that of [4]. We assume there is a finite excessive reference measure denoted $m$. The resolvent is denoted $U^{\lambda}, \lambda \geqq 0$. By $P^{m}$ we mean the measure $\int P^{x}() m(d x)$.

A non-negative function $s$ is called excessive if

$$
\sup _{t>0} P_{t} s=\lim _{t \rightarrow 0} P_{t} s=s
$$

where $P_{t}$ is the transition semi-group of the process. An excessive function $s$ is called a class $(D)$ potential if

$$
\lim P_{T_{n}} s=0 \quad \text { a.e. }
$$

whenever the sequence $T_{n}$ of stopping times increases to infinity.

Given a class $(D)$ potential $s$, we can write

$$
s\left(X_{t}\right)=E^{\cdot}\left[A(\infty) \mid \mathscr{F}_{t}\right]-A_{t}
$$

where $A$ is a natural additive functional defined off a polar set [14]. And

$$
E^{\cdot}\left[A^{2}(\infty)\right]
$$

is itself a class $(D)$ potential provided it is finite almost everywhere.

To each class $(D)$ potential $s$ we can associate a non-negative number, called its mass functional $L$, defined by

$$
L(s)=\lim _{t \rightarrow 0}\left(\frac{s-P_{t} s}{t}, 1\right) .
$$

In [14] a class $(D)$ potential $s$ was termed of finite energy if

$$
L\left(E^{\cdot}\left(A^{2}(\infty)\right)\right)<\infty
$$

and the square root of this number the energy of $s$. Up to a constant factor this agrees with the concept of energy in the classical sense. 
The above discussion remains intact if we take differences of class (D) potentials. The seminorm so obtained will be denoted $\|s\|_{e}$.

For symmetric processes another definition of energy is given in [8]: A function $u$ such that $u(X)$ is cadlag is of finite energy if

$$
\lim _{t \rightarrow 0} \frac{1}{2 t} E^{m}\left[\left(u\left(X_{t}\right)-u\left(X_{0}\right)\right)^{2}\right]
$$

exists and is finite. Its value is termed the square of the energy of $u$.

We shall now see how to relate these definitions with the definition of energy of the cadlag process $s(X)$ studied in Section 1.

Let us introduce some convenient notation. If $f$ is a Borel function and $\mu$ a measure $(f, \mu)$ will denote $\int f d \mu$. If $\mu=g d m$ for some $g$ we simply write $(f, g)$.

For any Borel function $f$ such that $f(X)$ is cadlag we shall write

$$
S(n, x, f(X))
$$

when the $S$ in (1) of Section 1 is evaluated relative to $P^{x}$. We just write $S(n, f(X))$ if it is evaluated relative to $P^{m}$.

Proposition 1. Let $X$ have a strong Markov dual $\hat{X}$ and assume that the expected life time $\hat{E}^{\cdot}[\hat{\xi}]$ of $\hat{X}$ is bounded. Let $u \in L^{2}(m)$ and suppose that $u(X)$ is cadlag. If

$$
\frac{1}{t} E^{m}\left[\left(u\left(x_{t}\right)-u\left(x_{0}\right)\right)^{2}\right] \leqq a<\infty
$$

then $u(X)$ has finite energy relative to $P^{m}$.

Proof. Fixing $n$, put

$$
g(x)=\sum_{1}^{2^{n}-1} E^{x}\left[\Delta^{2}(n, i, u(X))\right] .
$$

Then using the excessivity of $m$ and Markov property we get

$$
(g, 1) \leqq a
$$

where $a$ is a bound for the quantity in (3). And

$$
S(n, x, u(X))-u^{2}(x)=\sum_{0}^{\infty} P_{k} g .
$$

so that 


$$
S(n, u(X))=\left(u^{2}, 1\right)+\sum_{0}^{\infty}\left(g, \hat{P}_{k} 1\right) .
$$

Since $\sum_{0}^{\infty} \hat{P}_{k} 1 \leqq 1+\hat{E}^{\cdot}[\hat{\xi}]$, the result is thus clear from the hypothesis.

From Theorem 1 of Section 1, and the above proposition, relative to $P^{m}$, there is a decomposition

$$
u(X)=M+A .
$$

Since $A(0)=0, M(0)=u\left(X_{0}\right)$. We have

Proposition 2. With the above notation

$$
E^{m}\left[(M(t)-M(0))^{2}\right]+E^{m}\left[A^{2}(t)\right] \leqq 4 a t
$$

Proof. Just as in the proof of Proposition 1 it is seen that for any $i 2^{-k}$

$$
\sum_{j \leqq 2^{n-k_{i}-1}} E^{m}\left[\Delta^{2}(n, j, u(X)] \leqq \frac{a i}{2^{k}} .\right.
$$

Also for a subsequence $n_{p}$

$$
u\left(X_{0}\right)+\sum_{j \leqq 2^{n} n^{-k_{(i-1)}}} \delta\left(n_{p}, j, u(X)\right)
$$

converges weakly to $M\left(i / 2^{k}\right)$, where $\delta$ is defined in the proof of Theorem 1. Since $E^{m}\left[\Delta^{2}\right] \geqq E^{m}\left[\delta^{2}\right]$ we get from (6) and (7)

$$
\begin{aligned}
& E^{m}\left[\left(M\left(\frac{i}{2^{k}}\right)-M(0)\right)^{2}\right] \leqq \lim \text { inf of left side of (5) } \\
& \text { as } n \text { ranges through } n_{p} \text { is less or equal to } \frac{a i}{2^{k}} .
\end{aligned}
$$

From this to get (5) is easy.

Let now $s$ be square summable and a difference of class $(D)$ potentials with $\|s\|_{e}<\infty$. Write

$$
s(x)=E^{\cdot}\left[A(\infty) \mid F_{t}\right]-A_{t}
$$

We want to compare $\|s\|_{e}^{2}$ with

$$
\left.\|s\|_{e}^{2}=\lim _{t \rightarrow 0} \frac{1}{t} E^{m}\left[\left(s\left(X_{t}\right)\right)-s\left(X_{0}\right)\right)^{2}\right] .
$$

We shall soon see that this limit exists.

It is easy to see that 


$$
E^{\cdot}\left[A^{2}(\infty)\right]-s^{2}(x)
$$

is excessive. The mass functional being monotone $L\left(E^{\cdot}\left(A^{2}(\infty)-s^{2}\right)\right.$ is also finite. From the definition of mass functional we then see that

$$
\lim _{t \rightarrow 0}\left(s^{2}, \frac{1-\hat{P}_{t} 1}{t}\right)
$$

exists and is finite. Here $\hat{P}_{t}$ is just the adjoint of $P_{t}$ as an operator in $L^{1}$.

Let $n \geqq k$ be integers, fixed for the moment. Put for simplicity in notation

$$
\varphi=E \cdot\left[\left(s\left(X_{1 / 2 n}\right)-s\left(X_{0}\right)\right)^{2}\right]
$$

and $D$ the operator

$$
D=P_{1 / 2^{n}}
$$

Then

$$
D^{2 n-k}=P_{1 / 2^{k}}, \quad S(n, \cdot, s(X))=s^{2}+\sum_{0}^{\infty} D^{i} \varphi .
$$

Since $s$ is assumed to be square summable and a difference of potentials of finite energy, the following computations are legitimate.

$$
\begin{aligned}
2^{k}\left(S(n, \cdot, s(X)), 1-\hat{P}_{1 / 2^{k}} 1\right) & =2^{k}\left(s^{2}, 1-\hat{P}_{1 / 2^{k}} 1\right)+2^{k} \sum_{0}^{2^{n-k}-1}\left(D^{i} \varphi, 1\right) \\
& \leqq 2^{k}\left(s^{2}, 1-\hat{P}_{1 / 2^{k}} 1\right)+2^{n}(\varphi, 1)
\end{aligned}
$$

(by excessivity of $m$ ).

Now the variable $A(\infty)$ being the weak limit in $L^{2}$ of the variables

we have

$$
s\left(X_{0}\right)+\sum_{i}\left[s\left(X_{(i+1) / 2^{n}}\right)-E^{\cdot}\left(s\left(X_{(i+1) / 2^{n}}\right) \mid F_{i / 2^{n}}\right)\right]
$$

$$
E^{\cdot}\left[A^{2}(\infty)\right] \leqq \lim _{n} \inf S(n, \cdot, s(X)) .
$$

Thus, using the definition of mass functional, (9) and (10) we have proved part of

Theorem 3. Let $s \in L^{2}$ be a difference of class $(D)$ potentials of finite energy. Then

$$
\begin{aligned}
\|s\|_{e}^{2} & \leqq \lim _{t \rightarrow 0}\left(s^{2}, \frac{1-\hat{P}_{t} 1}{t}\right)+\|s\|_{e}^{2} \\
& =2 \lim _{t \rightarrow 0}\left(s, \frac{s-P_{t} s}{t}\right)
\end{aligned}
$$


where $\|s\|_{e}^{2}$ is defined in (8).

Proof. First we prove the existence of the limit following equality sign in (11). By assumption $s$ is a difference of class $(D)$ potentials of finite energy. If $\gamma$ is a class $(D)$ potential of finite energy $U\left(\left(\gamma-P_{t} \gamma\right) / t\right)$ increases to $\gamma$. It is proved in Theorem 1.4 of [14] that these potentials then converge "weakly in energy" to $\gamma$. Applying this to differences we deduce that

$$
\lim _{t \rightarrow 0}\left[\left(s, \frac{s-P_{t} s}{t}\right)+\left(\mu, U \frac{s-P_{t} s}{t}\right)\right]=\|s\|_{e}^{2}
$$

where $\mu$ is the Revuz measure of $s$ [15]. By dominated convergence

$$
\lim _{t \rightarrow 0}\left(\mu, U \frac{s-P_{t} s}{t}\right)=\lim _{t \rightarrow 0} \frac{1}{t}\left(\mu, \int_{0}^{t} P_{v} s d v\right)=(\mu, s)
$$

By Meyer's energy formula [11]

$$
E^{\cdot}\left[A^{2}(\infty)\right]=E \cdot\left[\int_{0}^{\infty} s\left(X_{t}\right)_{-} d A_{t}\right]+E \cdot\left[\int_{0}^{\infty} s\left(X_{t}\right) d A_{t}\right]
$$

Applying the mass functional $L$ to the above formula and using (12) and (13) we get

$$
\lim _{t \rightarrow 0}\left(s, \frac{s-P_{t} s}{t}\right)=L\left\{E \cdot\left[\int_{0}^{\infty} s\left(X_{t}\right)_{-} d A_{t}\right]\right\}
$$

Now for any $t$ we have

$$
\left(s^{2}, 1-\hat{P}_{t} 1\right)+E^{m}\left[\left(s\left(X_{t}\right)-s\left(X_{0}\right)\right)^{2}\right]=2\left(s, s-P_{t} s\right) .
$$

Using (9) thus the limit in (8) exists and the proof is complete.

Corollary 4. Let $s$ be as in Theorem 3. Then

$$
\|s\|_{e}^{2}=2 \lim _{t \rightarrow 0}\left(s, \frac{s-P_{t} s}{t}\right)
$$

if and only if $s$ is regular i.e. the corresponding additive functional $A$ is continuous.

Proof. If $s$ is as in Theorem 3, the difference of the right and left sides of (15) is just the mass functional applied to the excessive function.

$$
E^{\cdot}\left[\int_{0}^{\infty}\left(A_{t}-A_{t_{-}}\right) d A_{t}\right]
$$


This is immediate from (14) and Meyer's energy formula.

Remark. If $s$ is as in Theorem 3, it is easy to see that

$$
\lim _{n} S(n, x, s(X))=E^{x}\left[A^{2}(\infty)\right]+E^{x}\left[\int_{0}^{\infty}\left(A_{t}-A_{t-}\right) d A_{t}\right]
$$

for all $x$ such that $E^{x}\left[A^{2}(\infty)\right]<\infty$. On the other hand for any square integrable $u$ such that $u(X)$ is cadlag and has finite energy relative to $P^{m}$ the function

$$
\lim _{n} \inf \left\{S(n, \cdot, u(X))-u^{2}\right\}
$$

is super-median.

\section{§3. Special cases}

\section{Symmetric processes}

Consider now the classical case. Thus the state space is a bounded open subset $D$ of $\boldsymbol{R}^{d}, d \geq 3$, and $X$ is the Brownian motion killed at the exit time from $D$.

Symmetry of the transition kernel easily implies that for any $f \in L^{2}(D)$,

$$
\left(f, \frac{f-P_{t} f}{t}\right)
$$

is decreasing in $t$. Indeed, by density it is sufficient to verify this for $f \in \mathscr{D}(A)$ domain of generator $\mathscr{A}$ of $P_{t}$ regarded as a semigroup on $L^{2}(D)$. Now if $f \in \mathscr{D}(\mathscr{A})$,

$$
\begin{aligned}
\frac{d}{d s}\left(f, P_{s} \mathscr{A} f\right) & =\frac{d}{d s}\left(\mathscr{A} f, P_{s} f\right)=\left(\mathscr{A} f, P_{s} f\right) \\
& =\left(P_{s / 2} \mathscr{A} f, P_{s / 2} \mathscr{A} f\right) \geq 0
\end{aligned}
$$

so that $\left(f, P_{s} \mathscr{A} f\right)$ is increasing in $s$. But then the same is true of

$$
\frac{1}{t} \int_{0}^{t}\left(f, P_{s} \mathscr{A} f\right) d s=\left(f, \frac{P_{t} f-f}{t}\right) .
$$

In other words the quantity in (3.1) is decreasing. Thus the quantity in (3.1) is bounded in $t$ if and only if the limit as $t$ tends to zero is finite.

Corollary 4 of Section 2 is certainly valid for the Brownian motion because all excessive functions are regular. Also every class $(D)$ potential $s$ such that $\|s\|_{e}<\infty$ is necessarily in $L^{2}(D)$. Completion of this space 
with the energy norm is usually called the space of BLD functions or the Sobolev space $\mathscr{H}_{0}^{\prime}$. Thus if $f \in \mathscr{H}_{0}^{\prime}$

$$
\|f\|_{e}^{2}=2 \lim _{t \rightarrow 0}\left(f, \frac{f-P_{t} f}{t}\right) .
$$

It is known that if $f \in \mathscr{H}_{0}^{\prime}, f(X)$ is continuous relative to $\boldsymbol{P}^{m}$. By (3.2) and Proposition 1 of Section $2 f(X)$ has finite energy. We can write by Theorem 1 of Section 1,

$$
f(X)=M+A
$$

Let us show that this decomposition is unique. By Corollary 2 of Section 1 it is sufficient to show that the quadratic variation $Q(A)=0$ and this is immediate from Theorem 3, Section 1 because the energy norm of the process $f(X)$ is dominated by $\|f\|_{e}$ as shown in Proposition 1 of Section 2.

Note. It is clear that no special properties of the Brownian motion process are used in the above discussion.

About characterisation of $\mathscr{H}_{0}^{\prime}$

As above $D$ will be a bounded open subset of $\boldsymbol{R}^{n}$ and $\mathscr{H}_{0}^{\prime}=\mathscr{H}_{0}^{\prime}(D)$ the space of BLD functions on $D$. We now give a local characterisation of this space in terms of its composition with the Brownian motion. Before we do this we need the following simple proposition which classifies the role of (3.1) in these considerations.

Proposition 1. For $f \in L^{2}(D)$ let $\|f\|_{t}$ denote the quantity in (3.1) with $\|f\|_{0}=\lim _{t \rightarrow 0}\|f\|_{t}$. Then

$$
\begin{aligned}
& \left\|P_{h} f\right\|_{t} \leq\|f\|_{t} \text { for all } t, h>0 \\
& \left\|f-P_{h} f\right\|_{0} \rightarrow 0 \text { as } h \rightarrow 0 \text { if }\|f\|_{0}<\infty .
\end{aligned}
$$

Finally

$$
f \in \mathscr{H}_{0}^{\prime} \text { if and only if }\|f\|_{0}<\infty \text {. }
$$

Proof. The proof given above that $\|f\|_{t}$ is decreasing also shows that

$$
\left(f, \frac{P_{s} f-P_{t+s} f}{t}\right)
$$

is decreasing both in $s$ and $t$. (3.3) is then immediate upon replacing $s$ in (3.5) by $2 h$. To prove (3.4) first choose $s$ so that

$$
\|f\|_{0}-\|f\|_{s}<\varepsilon
$$


for a given $\varepsilon>0$. Then for any $t \leq s$,

$$
\begin{aligned}
\left\|f-P_{h} f\right\|_{t} & =\|f\|_{t}-2\left(f, \frac{P_{h} f-P_{t+h} f}{t}\right)+\left\|P_{h} f\right\|_{t} \\
& \leqq\|f\|_{t}-\left(f, \frac{P_{h} f-P_{t+h} f}{t}\right) \\
& \leqq \varepsilon+\|f\|_{s}-\left(P_{h} f, \frac{f-P_{s} f}{s}\right) \leqq 2 \varepsilon
\end{aligned}
$$

for small enough $h$.

The final statement is immediate from (3.4) because $P_{h}$ maps $L^{2}(D)$ into $\mathscr{D}(\mathscr{A}) \in \mathscr{H}_{0}^{\prime}$.

The following remark may be helpful.

Remark. Proposition 1 of Section 2 shows that the energy of Process $f(X)$ with $f \in L^{2}(D)$ is connected with the quantity in (2.8). It is easily seen that $\|f\|_{0}$ as defined above equals

$$
\lim _{t \rightarrow 0} 2^{n} E^{m}\left[\left(f\left(X_{2-n}\right)-f\left(X_{0}\right)\right)^{2}\right]
$$

provided

$$
\lim _{t \rightarrow 0} \frac{1}{t}\left(f^{2}-P_{t} f^{2}, 1\right)=0
$$

And this last limit does obtain provided $f$ has compact support in $D$.

With these details out of the way we can now state

TheOREm 2. Let $f \in L^{2}(D)$ have compact support in $D$. Then $f \in \mathscr{H}_{0}^{\prime}$ if and only if $f(X)$ is cadlag and has finite energy relative to $P^{m}$.

Proof. The if part has already been shown. Suppose $f$ satisfies the hypotheses of the theorem. Fix $n$ and put

$$
g=E^{x}\left[\left(f\left(X_{2-n}\right)-f\left(X_{0}\right)\right)^{2}\right] .
$$

We compute, $\tau$ denoting the first exit time from $D$

$$
\begin{aligned}
S(n, f(X))-\left(f^{2}, 1\right) & =\sum_{0}^{\infty}\left(P_{i 2-n} q, 1\right)=\left(g, \sum_{0}^{\infty} P_{i 2-n} 1\right) \\
& =\left(g, \sum_{0}^{\infty} P^{\cdot}\left(\tau>i 2^{-n}\right)\right) \geqq 2^{n}\left(g, \int_{0}^{\infty} P^{\cdot}(\tau>t) d t\right) \\
& =2^{n}\left(g, E^{\cdot}\left(\tau^{2}\right)\right)
\end{aligned}
$$


Let $V \subsetneq D$, be a relatively compact neighbourhood of the support of f. On $V, E^{\cdot}\left(\tau^{2}\right)$ is bounded below by say $a$. Then denoting by $Y$ the process killed at exit from $V$,

$$
2^{n} E^{m}\left[\left(f\left(Y_{2-n}\right)-f\left(Y_{0}\right)\right)^{2}\right] \leqq 2^{n} \int_{V} g d m \leqq 2^{n} \frac{1}{a}\left(g, E^{\cdot}\left(\tau^{2}\right)\right) \leqq S(n, f(X)) .
$$

Since $f$ has support in $V$, Proposition 1 together with Remark force the conclusion

$$
f \in \mathscr{H}_{0}^{\prime}(V) \subseteq \mathscr{H}_{0}^{\prime}(D)
$$

That concludes the proof.

\section{Sector condition}

Now we consider very briefly a slightly more general situation.

This condition states: For all measurable $f$ and $g$ whose potentials $U f$ and $U g$ have finite energy we have

$$
|(U f, g)| \leqq M\|U f\|_{e}\|U g\|_{e}
$$

for some constant $M>0$. To our knowledge this condition appears for the first time in these considerations in M. Silverstein [17].

According to [14] the set of potentials $U f$ of finite energy is weakly dense in energy in the space of all class $(D)$ potentials of finite energy. So if (3.6) obtains, then

$$
|(s, g)| \leqq M\|s\|_{e}\|U g\|_{e}
$$

It is also known that under the sector condition all excessive functions are regular.

Let us assume one more condition: For all $t>0$

$$
\left\|P_{t} U f\right\|_{e} \leqq M\|U f\|_{e} .
$$

It is easily seen using Fourier transforms that (3.8) holds for all Lévy processes, with $M=1$.

Now let $s$ be a difference of class $(D)$ potential of finite energy. For $t>0$, the potential of $1 / t\left(s-P_{t} s\right)$ is

$$
\frac{1}{t} \int_{0}^{t} P_{u} s d u
$$

with energy 


$$
\left\|\frac{1}{t} \int_{0}^{t} P_{u} s d u\right\|_{e} \leqq \frac{1}{t} \int_{0}^{t}\left\|P_{u} s\right\|_{e} d u \leqq M\|s\|_{e}
$$

We are of course using (3.8). And using (3.7)

$$
\left(s, \frac{s-P_{t} s}{t}\right) \leqq M^{2}\|s\|_{e}^{2} .
$$

Now we can argue as in the symmetric case and conclude: If a Borel function $f$ is a limit of a sequence $s_{n}$ of differences of class $(D)$ potentials which is Cauchy in energy and if $f(X)$ is cadlag then we can write

$$
f(X)=M+A
$$

uniquely with $M$ a martingale and $A$ previsible of zero quadratic variation.

\section{REFERENCES}

[1] C. Berg and G. Forst, Non-symmetric translation invariant Dirichlet forms, Invent. Math., 21 (1973), 199-212.

[ 2 ] A. Beurling and J. Deny, Dirichlet spaces, Proc. Nat. Acad. Sci. U.S.A., 45 (1959), 208-215.

[ 3 ] J. Bliedner, Functional spaces and their exceptional sets, Seminar on Potential Theory II, Lecture Notes in Math., 226, Springer, 1971.

[4] R. M. Blumenthal and R. K. Getoor, Markov processes and potential theory, Academic Press, 1968.

[5] G. A. Brosamler, Quadratic variation of potentials and harmonic functions, Trans. Amer. Math. Soc., 149 (1970), 243-257.

[6] H. Cartan, Sur les fondements de la théorie du porential, Bull. Soc. Math. France, 691 (1941), 71-96.

[ 7 ] C. Dellacherie and P. A. Meyer, Probabilities and Potential, North Holland, 1978.

[8] M. Fukushima, Dirichlet forms and Markov processes, North Holland/Kodansha, 1980.

[ 9 ] H. Föllmer, Calcul d'ITO sans probabilités, Séminaire de Probabilitiés XV, Lecture Notes in Math., 850, Springer, 1981.

[10] S. E. Graversen and M. Rao, On a Theorem of Cartan, Preprint Series 33, 1983/84, Aarhus University.

[11] P. A. Meyer, Probability and Potentials, Blaisdell, Massachusetts, 1966.

[12] — Un cours sur les intégrales stochastiques, Séminaire de Probabilitiés X, Lecture Notes in Math., Springer, 511 (1976), 245-400.

[13] —- Le dual de $H^{1}\left(\boldsymbol{R}^{\nu}\right)$ Séminaire de Probabilités XI, Lecture Notes in Math., Springer, 581 (1977), 132-195.

[14] Z. R. Pop-Stōjanovic and M. Rao, Convergence in Energy, Z. Wahrsch. Verw. Gebiete, 69 (1985), 593-608.

[15] D. Revuz, Mesures associées aux fonctionelles additives de Markov I, Trans. Amer. Math. Soc., 148 (1979), 501-531.

[16] M. L. Silverstein, Symmetric Markov processes, Lecture Notes in Math., 426, Springer, 1974. 
[17] M. L. Silverstein, The sector condition implies that semi-polar sets are quasipolar, Z. Wahrsch. Verw. Gebiete, 41 (1977), 13-33.

[18] T. Yamada and Y. Oshima, On some representations of continuous additive functionals locally of zero energy, J. Math. Soc. Japan, 36 (1984), 315-339.

Department of Mathematics

Arhus University

Ny Munkegade

8000 Arhus $C$

Denmark 